\title{
Las huertas periurbanas del mediterráneo (Murcia-Alicante-Valencia y Zaragoza). Primeros resultados de investigación para el caso de Valencia.
}

\author{
Rafael R. Temes Córdovez ${ }^{1}$, Clara García Mayor², Juan J. Tuset Davóz, \\ Ana Ruiz Varona ${ }^{4}$, Fernando García Martín ${ }^{5}$, Marcos Ros Sempere ${ }^{5}$ \\ ${ }^{1}$ Departamento de Urbanismo, Universidad Politécnica de Valencia, Valencia, ${ }^{2}$ Departamento \\ de Edificación y Urbanismo, Universidad de Alicante, Alicante, ${ }^{3}$ Departamento de Proyectos \\ Arquitectonicos, Universidad Politécnica de Valencia, Valencia, ${ }^{4}$ Escuela de Arquitectura y \\ Tecnología, Universidad San Jorge, Zaragoza, ${ }^{5}$ Departamento de Arquitectura y Tecnología de la \\ Edificación, Universidad Politécnica de Cartagena, Cartagena, España. \\ E-mail: 'rtemesc@urb.upv.es,
}

\begin{abstract}
Resumen. Las Huertas históricas periurbanas de regadio tradicional han sido símbolo de la identidad del paisaje, la economía y la cultura de algunas cidudades mediterráneas. Por su propia naturaleza, han sido históricamente un sistema de regulación de las condiciones ambientales primigenias de las llanuras aluviales en las que se emplazan - eliminación de espacios palustres insalubres, regulación de episodios de inudación, mejora de las condiciones en los periodos de largas sequías-. En la actualidad sólo quedan algunos reductos de este paisaje en las periferias urbanas, principalmente en España. En un momento en que la planificación de las ciudades del siglo XXI busca integrar objetivos y estrategias para la sostenibilidad ambiental y social, estas huertas periurbanas aglutinan características únicas y se presentan como un ejemplo de integración del paisaje productivo con la mejora de la calidad de vida de los entornos urbanos en que se insertan. Sometidas a fuertes presiones urbanísticas, este trabajo recoge los primeros pasos en la consolidación de un grupo de investigación centrado en la mejor comprensión y parametrización de estos espacios únicos, orientados a aportar estrategias que incidad en la sostenibilidad ambiental y social de los contextos urbanos en los que se integran.
\end{abstract}

\author{
Palabras clave: Huerta, paisaje cultural, sostenibilidad \\ medioambiental, invariantes.
}

\section{Introducción}

Las Huertas periurbanas poseen una gran importancia estratégica relacionada con la producción de alimentos (Beltrán, 2012), prestación de servicios ecosistémicos (Elmqvist, et al., 2015) así como un elevado valor histórico patrimonial debido a sus complejos sistemas de regadío tradicionales (Glick, 1970; Burriel, 1971; Courtot, 1986). Además, su posición de frontera con el continuo urbano hace especialmente vulnerable esta franja en la que las tensiones de ocupación y continuo cambio han sido la tónica dominante (Temes, 2016; Ros \& García-Martín, 2016; García-Mayor \& Canales, 2018). Como comenta Romero (2016), la (des)protección de las grandes Huertas mediterráneas periurbanas españolas y la ausencia de iniciativas políticas de coordinación a escala metropolitana sintetizan muy bien el riesgo que supone carecer de mecanismos coherentes eficaces de ordenación y gestión en la segunda década del siglo XXI. Las huertas mediterráneas representan un tipo de paisaje de gran singularidad por su rareza tipológica en Europa, como reconoce 
el Informe Dobris de la Agencia Europea de Medio Ambiente (1998), que sólo identifica 6 paisajes de este tipo en Europa. Como define la UNESCO, el paisaje cultural, denominación en la que se inscriben los paisajes de la huerta, son una realidad compleja, integrada por componentes naturales y culturales, tangibles e intangibles, cuya combinación configura el carácter que lo identifica como tal.

En la línea de trabajar sobre la naturaleza de los cambios y transformaciones que han ido experimentando los reductos de Huertas Históricas Periurbanas del Mediterráneo (HHPM) que podemos identificar aún en España, en la franja mediterránea del Levante y Aragón, hemos formado una Red de Investigadores comprometidos con el análisis y estudio de estos reductos de HHPM que han configurado la identidad económica y cultural de las comarcas en las que se desarrollaron, así como los cambios experimentados en este paisaje desde la perspectiva de la sostenibilidad ambiental y social. Prácticamente en paralelo, desde los diferentes grupos de investigación que constituyen hoy la Red, hemos ido estudiando los cambios y evolución de nuestros reductos de HHPM. En Murcia a través del Laboratorio de Investigación Urbana de la Universidad Politécnica de Cartagena, en el año 2015 dentro del Proyecto competitivo "Migraciones contemporáneas" (15235/PPC10), se inició un primer trabajo de análisis sobre la Huerta de Murcia (Ros \& García, 2016a) que derivó en varias publicaciones posteriores (Ros y García, 2016b, 2017) y exposiciones itinerantes. En Alicante, la investigación doctoral de GarcíaMayor (2015)(García-Mayor, 2015) sobre la Huerta de Orihuela en la Vega Baja del río Segura marca una sólida línea de trabajo entorno a la comprensión de los elementos constitutivos de la estructura del paisaje de huerta, con el objetivo de elaborar una caracterización en base a los elementos invariantes que definen la espacialidad de este paisaje agrícola periurbano (García-Mayor, 2017; García Mayor \& Canales Martínez, 2015) así como la parametrización de la evolución del área de estudio (Garcia-Mayor \& Canales Martínez, 2018-a; García-Mayor \& Canales Martinez, 2018-b). En Valencia los trabajos atienden a dos líneas de trabajo. Por un lado, aquella que trata de medir y cuantificar los cambios experimentados en la huerta periurbana de la ciudad de Valencia (Temes \& Moya, 2015;2016) y por otro aquellos que indagan en la naturaleza de los paisajes multifuncionales de la huerta de Valencia (Tuset, 2018). En el caso de Zaragoza los trabajos se centran en el diseño de modelos de análisis espacial para la evaluación, en términos de vulnerabilidad y potencial revitalización, del espacio transformado de huerta periurbana (Ruiz, 2019).

A partir de estas aportaciones, se ha consolidado la Red de Investigación ${ }^{1}$ que ha organizado dos Jornadas de Investigación ${ }^{2}$ en las que se ha ofrecido los primeros resultados vinculados a la metodología de trabajo y análisis y en torno a la discusión de los temas prioritarios de investigación sobre estas huertas, que fueron presentados en el Congreso BIA Urban Regeneration Forum celebrado en Bilbao, España, en Mayo de 2018 (García-mayor, Miguel, Martín, \& Sempere, 2018; Tuset, Temes, \& Ruiz, 2018). El trabajo presentado en esta ponencia, trata de ofrecer de manera ordenada, noticia sobre los métodos de trabajo que se están utilizando para realizar un análisis comparativo de los cambios experimentados en las diferentes realidades de la huerta, así como exponer las líneas de trabajo en las que se está avanzando. Se trata aún de un trabajo inicial y unos primeros resultados que nos permitirán trabajar con objetivos más ambiciosos en una siguiente fase. Para ejemplificar mejor dichos avances, se expondrá con mayor detalle los trabajo elaborados para la Huerta de Valencia.

\section{Metodología de análisis para valorar las trasformaciones}

La forma y dimensión de las HHPM que estudiamos son muy diferentes y esto se refleja también en la naturaleza e intensidad de los cambios que han sido predominantes en cada caso. El ámbito estricto que define la huerta histórica de Valencia afecta a parte o a la totalidad de 40 municipios, abarcando una superficie aproximada de 23.000 ha de las que el área de suelo no urbanizable de huerta en regadío, es de una superficie de 11.393 ha. aproximadamente. 
Huerta histórica periurbana de Valencia Huerta histórica periurbana de Zaragoza
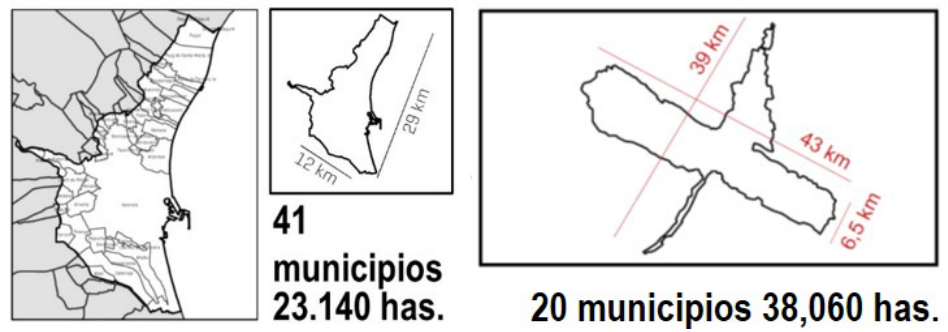

Fig. 1. Delimitación de las Huertas Periurbanas de Valencia y Zaragoza. 1957-2015. Fuente: Elaboración propia

Las huertas del Segura forman un continuo agrario compuesto por la Vega Media y la Huerta de Murcia en la Región de Murcia y la Vega Baja en Alicante. En la Región de Murcia tenemos que hablar de la existencia de 13 municipios que abarcan una superficie en zona de huerta de 21.169 has, muy próxima a la de Valencia. En el caso de la huerta de la Vega Baja de Alicante el número de municipios implicados es de 21, con una superficie de 710.398 has, de las que aproximadamente unas 21.000 has. corresponde a la huerta de regadío tradicional. En cambio, la delimitación de la huerta histórica de Zaragoza, que abarca 20 municipios, tiene un área de 38.060 ha, de las cuales 24.850 corresponden a la huerta de regadío tradicional (Figura 1 - Figura 2).

El método de análisis empleado en esta primera etapa, denominado "de coordinación cartográfico" (Temes, 2008), ha tenido el propósito de ser equivalente y compatible en todas las huertas para permitir una comparación adecuada de los distintos ámbitos de trabajo. El objetivo de medir los cambios producidos en dichos territorios se basa en la identificación de los siguientes elementos:

\section{Invariantes}

Estructura del territorio y el paisaje de las HHPM. Estará formado por los siguientes elementos:
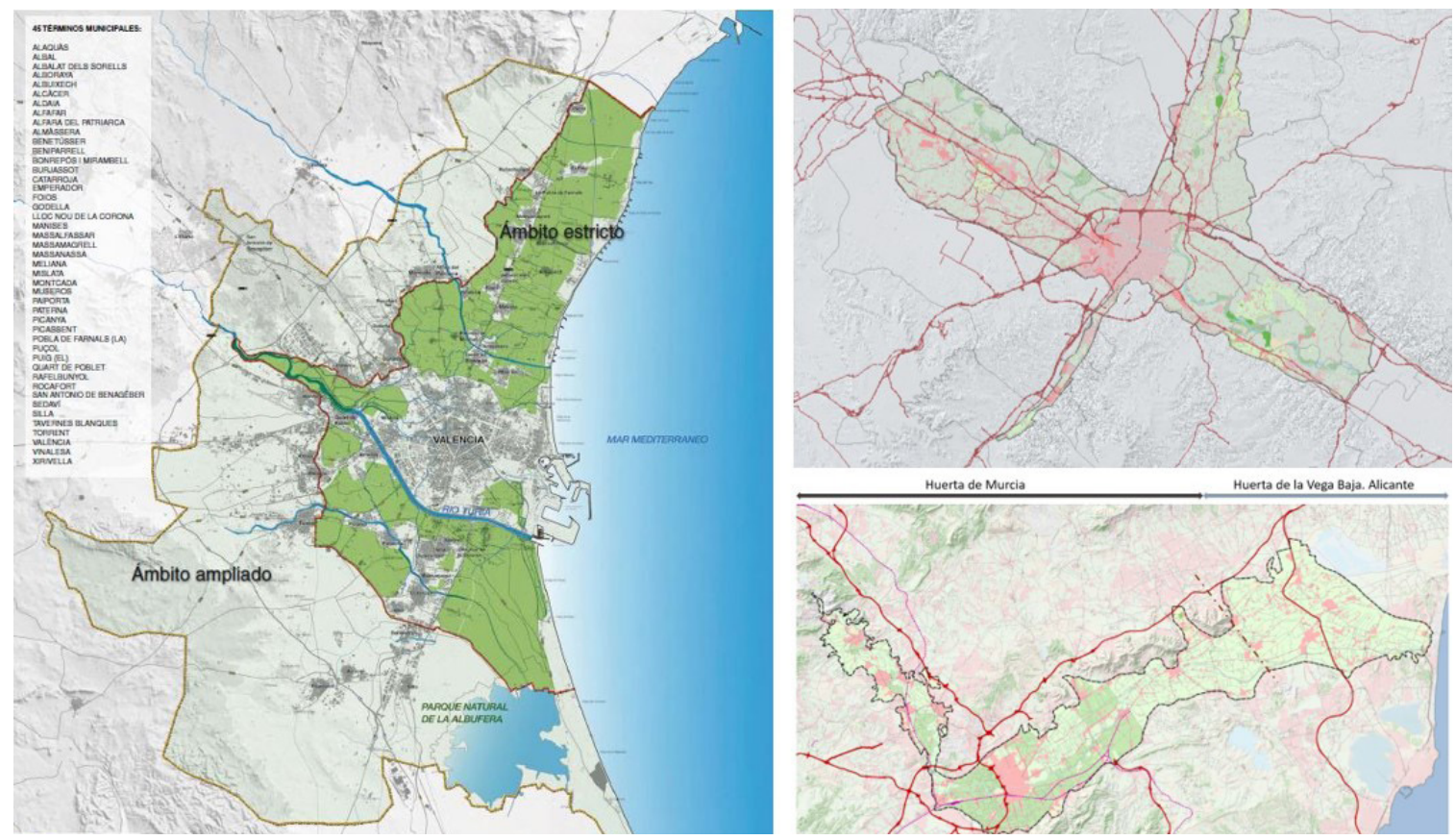

Fig. 2. A. Delimitación de las Huertas Periurbanas de Murcia y Vega Baja (Alicante). Es un mismo paisaje de Huerta pertenece a dos regiones administrativas independientes. Fuente: Elaboración propia B. Delimitación de la Huerta Periurbana de Valencia. C. Delimitación de la Huerta Periurbana de Zaragoza 


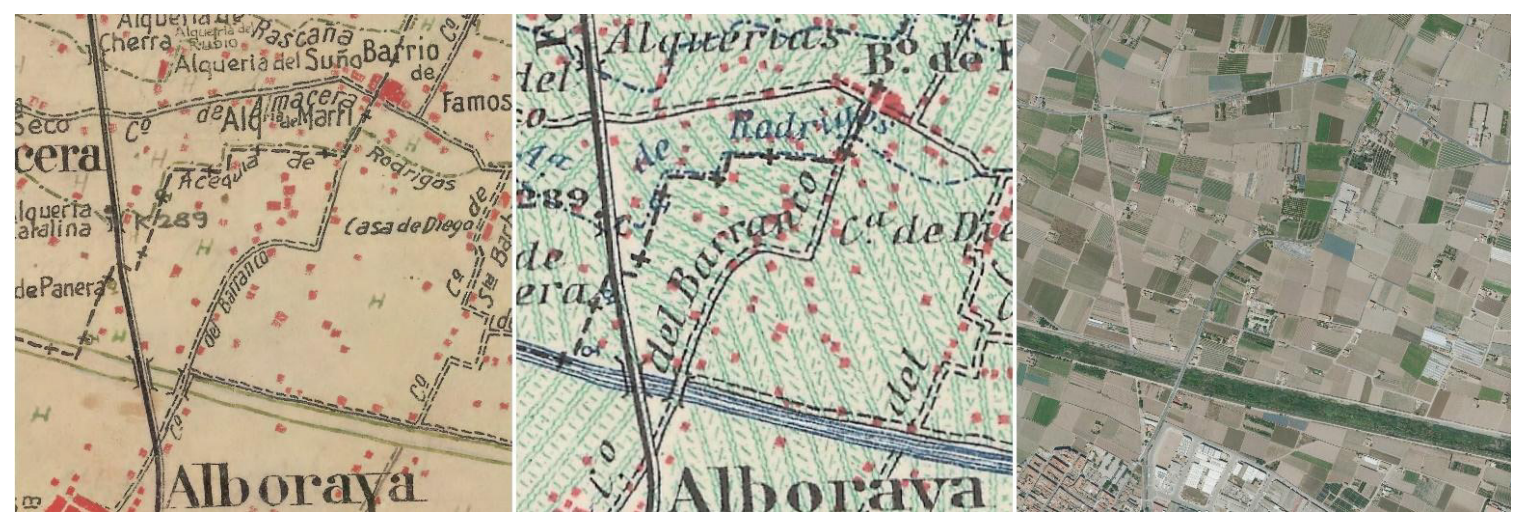

Fig. 3. Representación de caminos, acequias y cauces sobre las Minutas de referencia. Fuente: Elaboración propia

-Topografía, forma del territorio: modelo de sombra + curvas de nivel

-Red de riego y drenaje: Malla jerarquizada de canalizaciones de transporte de agua —Río, canales y acequias - y de drenaje de agua excedente - escorrentías, azarbes-

-Red de caminos, veredas y sendas: Malla jerarquizada de itinerarios que dan acceso al territorio y que discurren vinculados a la red de riego y drenaje.

-Posición de las poblaciones: En origen sobre los microrrelieves de las llanuras aluviales de la huerta, ocupando el mínimo espacio fértil.

Partiremos, para la representación e identificación de estos elementos, de las Minutas MTN50 del Instituto Geográfico Nacional (IGN) (1916-50) disponibles en los servicios WMS del IGN. Para la topografía y su identificación hemos trabajado con los MDT de paso de malla 5 metros del IGN (Figura 3).

Estructura parcelaria agrícola

Para valorar las permanencias y cambios en la estructura agrícola parcelaria, hemos empleado dos criterios. Desde 1945 hasta 2005 el análisis no lo podemos realizar a escala parcelaria pues no contamos con una fuente fiable que nos permita conocer la evolución de las estructuras agrícolas. En este caso el análisis, se hace de manera generalizada a partir de la identificación de cambios a partir de la comparación de las series de ortofotos georreferenciadas con los que contemos a partir de las fuentes del IGN. Las series seleccionadas, utilizando el caso de Valencia como ejemplo para mostrarlas son (Figura 4):
-VUELOAMERICANOSERIEA(1945/1946). Conjunto formado por 28 fotogramas

-VUELO INTERMINISTERIAL (1973/1986).

Conjunto formado por 53 fotogramas

-VUELO NACIONAL (1980/1986). Conjunto formado por 31 fotogramas

-VUELO QUINQUENAL (1993/2003). Conjunto formado por 32 fotogramas

Por otro lado, a partir de 2005 el método de análisis del parcelario agrícola lo hemos sustituido para ganar en exactitud. En este caso hemos empleado las series parcelaria provenientes del Sistema de Información Geográfica de Parcelas Agrícolas, (SIGPAC),

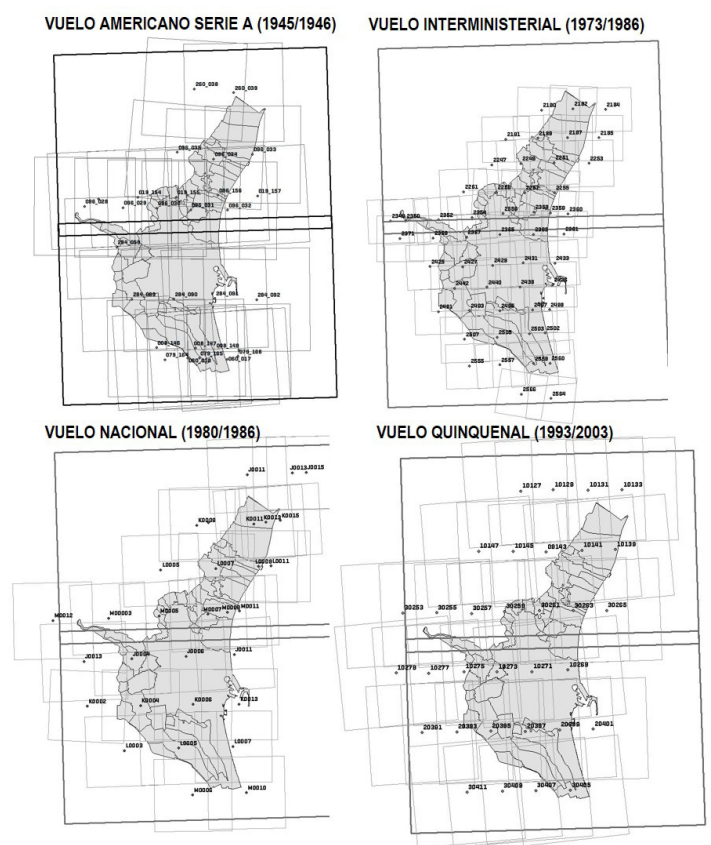

Fig. 4. Mapas de ubicación de fotogramas en cada vuelo seleccionado para el caso de la Huerta de Valencia. Fuente: Elaboración propia. 
que permite identificar geográficamente las parcelas declaradas por los agricultores $\mathrm{y}$ ganaderos y nos permite además hacer un análisis de los cambios de uso del suelo al menos durante los últimos 10 años.

\section{Crecimientos por extensión y dispersión en la huerta}

Los procesos de ocupación por parte de la edificación sobre los suelos de las HHPM los valoramos e identificamos dependiendo de su morfología. Para poder medir y comparar los crecimientos por extensión, producidos principalmente por el avance de los frentes urbanos de las poblaciones sobre la huerta, usaremos una representación vectorial con detalle de manzana extraída de la cartografía vectorial 1:50.000 del IGN, garantizando con ello la cobertura para todas las HHPM. La delimitación de la capa de ocupación se irá adaptando a cada período cronológico de estudio guiado por la referencia fotográfica con las que contamos (Figura 5).

Sin embargo, para los cambios producidos por los crecimientos por dispersión (diseminado y urbanizaciones), valoraremos dos técnicas diferentes de representación de su ocupación en planta:

-Representación simbólica (cuadrado). Partiremos de la representación vectorial que proviene originalmente de la cartografía vectorial 1:50.000 del IGN y añadiremos/ rectificaremos los elementos que comprobemos en la ortofoto de referencia. En este caso, sólo iremos poniendo un punto a modo de símbolo generalizado que representa la posición de una vivienda o construcción en la huerta. La nube de puntos que se generará se irá adaptando a cada período cronológico guiándose por la referencia fotográfica (Figura 6).

-Representación real redibujada sobre ortofoto (polígono). Partiremos de la representación vectorial que proviene originalmente de la cartografía catastral de rústica de la DGC y añadiremos/rectificaremos los elementos que

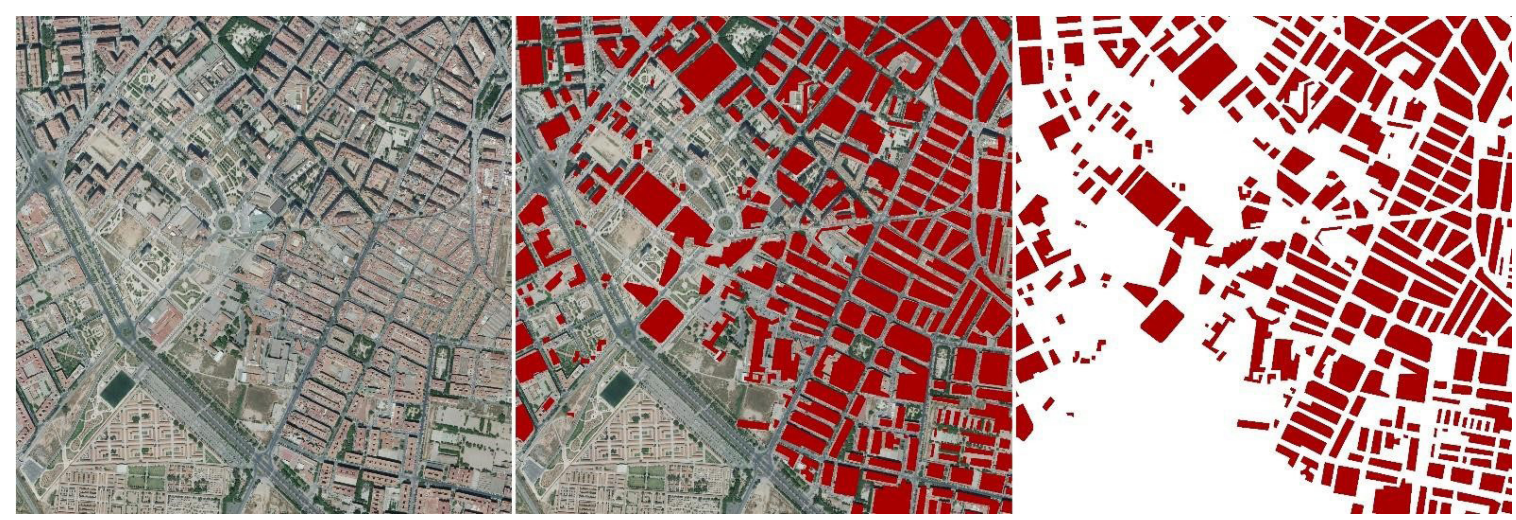

Fig. 5. Fotograma de referencia, superposición cartografía vectorial $\mathbf{5 0 . 0 0 0}$ IGN adaptación manual por observación. Fuente: Elaboración propia.

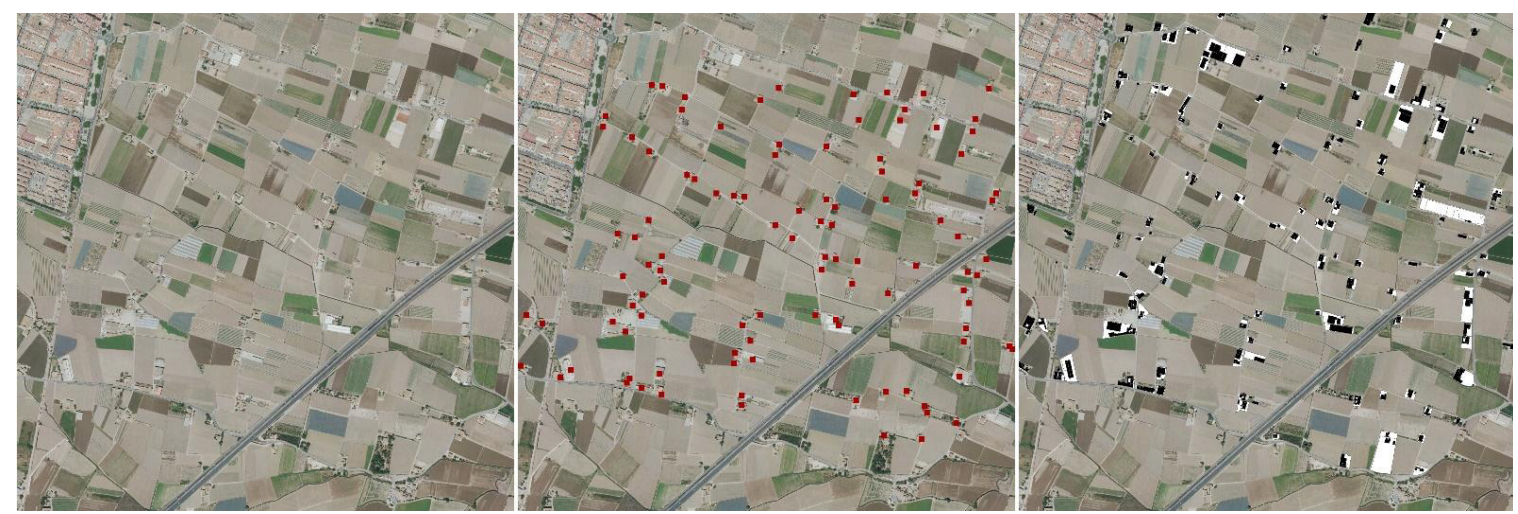

Fig. 6. Representación de dispersión mediante puntos (símbolos) o mediante cartografía catastral. Fuente: Elaboración propia. 
comprobemos en la foto. Dicha "mancha" se irá adaptando a cada período cronológico guiándose por la referencia fotográfica (Figura $6)$.

\section{Cambios de usos}

Más allá de las transformaciones físicas experimentadas en los invariantes, estructura parcelaria y edificación (disperso y continuo), en el proyecto necesitamos conocer los cambios de usos y ocupación que se han producido de manera general en el territorio. En los puntos anteriores hemos expuesto como mediante el SIGPAC podemos hacer un análisis suficientemente preciso para los usos agrícolas. Ahora bien, si queremos tener una valoración global de otros tipos de usos que también se dan dentro de los espacios de las huertas, es necesario emplear otro método. Para ello el trabajo se apoya en el Sistema de Información sobre Ocupación del Suelo de España (SIOSE) como fuente de información para medir los cambios de uso del suelo correspondiente con la delimitación de las HHPM. La serie temporal que podemos comparar se refieren a las fechas: 2005, 2011 y 2014.Inicialmente se han realizado la siguiente cartografía, definiendo las leyendas de la Figura 7:

1. Mapa 1, nivel 1

2. Mapa 2, nivel 2 artificial y cultivos

3. Mapa 3, nivel 4 artificial desagregado

4. Mapa 4, nivel 4 cultivos desagregado

Tras esta primera fase de trabajo se detectaron los siguientes problemas:

1. Las leyendas tienen una representatividad desigual para los cuatro casos de HHPM considerados, fundamentalmente en relación con el nivel 4 de cultivos desagregados. Los tipos de uso de suelo, especialmente los destinados a actividades agrícolas, son muy heterogéneos para los cuatro casos estudiados a pesar de utilizar teóricamente una misma metodología de identificación.

2. La ocupación de las HHPM por viviendas dispersas es un patrón de cambio significativo y que el estudio debe referir de manera específica. Sin embargo, el SIOSE lo incluye en la categoría urbano discontinuo/ urbano mixto, más genérica. En ocasiones la

$\square$ Delimitacion Huerta Vlc
Coberturas artificiales
Cultivos
Arbolado forestal
Matorral
Pastizal
Terrenos naturales sin vegetación
Coberturas de agua
Coberturas húmedas

Leyenda mapa 1

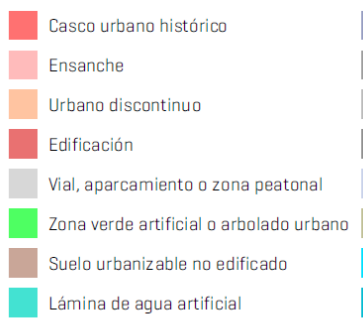

Leyenda mapa 3

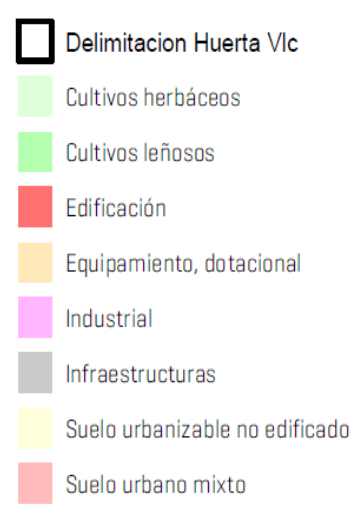

Leyenda mapa 2

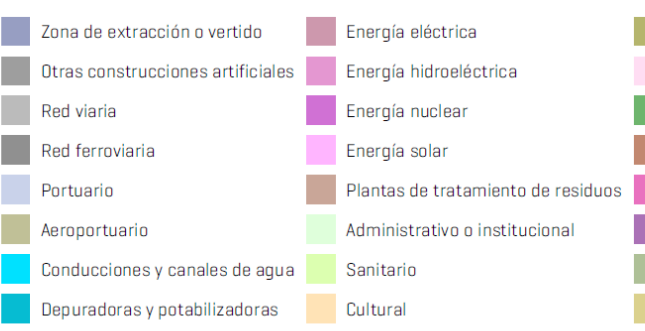

Leyenda mapa 4

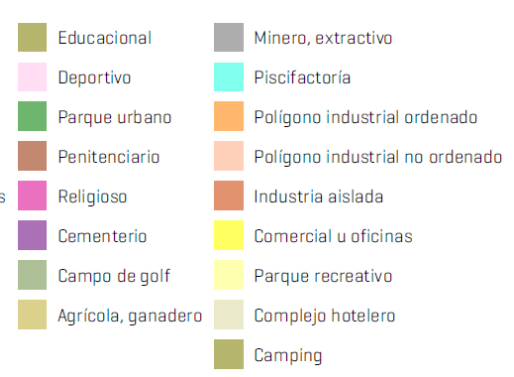

Fig. 7. Niveles de leyenda del SIOSE, 2011 utilizados en el proyecto de HHPM. 
huerta queda clasificada, de manera imprecisa, como cobertura artificial debido al disperso.

3. No existe un protocolo sencillo para evaluar la transformación de los usos de suelo de la capa de SIOSE durante estos últimos 10 años.

Teniendo en cuenta lo anterior, se propone:

1. Diseñar una clasificación que fuese representativa para el conjunto de HHPM y que refiera de manera específica a entornos periurbanos agrícolas e históricos, empleando para ello los porcentajes de cada uso en el polígono proporcionado por SIOSE,

a. Mejorando la representatividad de los cultivos desagregados (Nivel 4)

b. Eliminar clasificaciones erróneas de huerta como cobertura artificial correspondiente a la clasificación actual de zonas urbanas discontinuas.

c. Identificar los diferentes tipos de cubiertas que no han sido especificadas tras un cambio de uso del suelo.

2. Apoyo con otras fuentes de información espacial (en concreto, SIGPAC) como elemento que ayude a identificar las huertas en la proporción que ocupa en los polígonos del SIOSE de manera más exacta.

\section{Análisis de las transformaciones en el caso de la Huerta Histórica de Valencia.}

Cambios en la ocupación 1957 - 2015

La comparativa del período de estudio 19572015 , evidencia la gran transformación que ha sufrido la huerta de Valencia en este intervalo. Los datos que se deducen de la comparación gráfica son la de un crecimiento de la población de 781.113 en 1957 a 1.547 .479 en 2015 , lo que equivale al doble $(1,98 \%)$, que contrasta claramente con el crecimiento de la superficie del continúo edificado de 3.414 has. a 9.730 has., siendo un $2.85 \%$., casi unas tres veces más.

Al apreciar la "mancha" del continúo edificado y su transformación, se observa que en 1957 la estructura urbana de la ciudad de Valencia permite reconocer el centro histórico $\mathrm{y}$ los ensanches, algunos apéndices vinculados a caminos (crecimientos polarizados), el gran
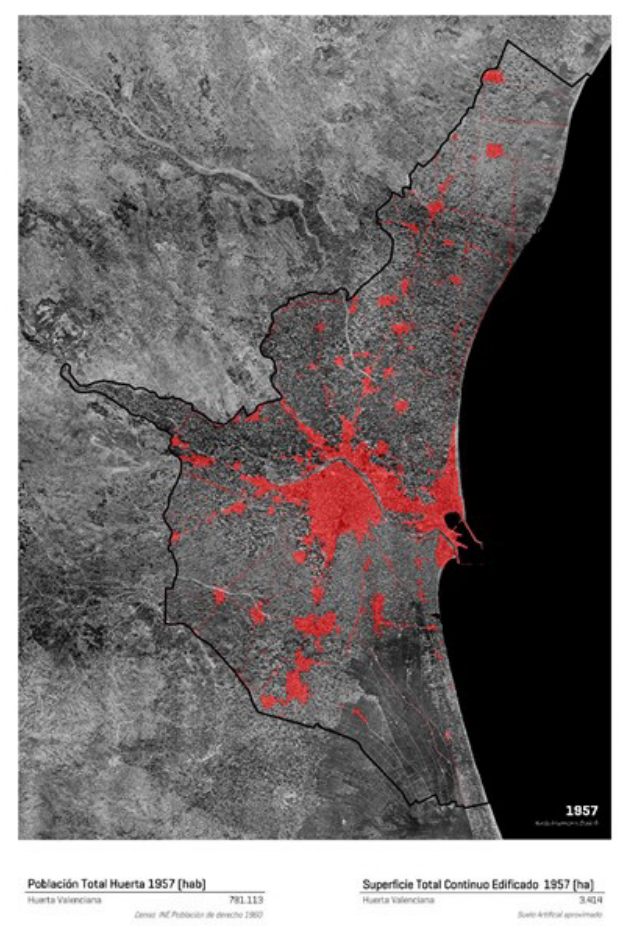

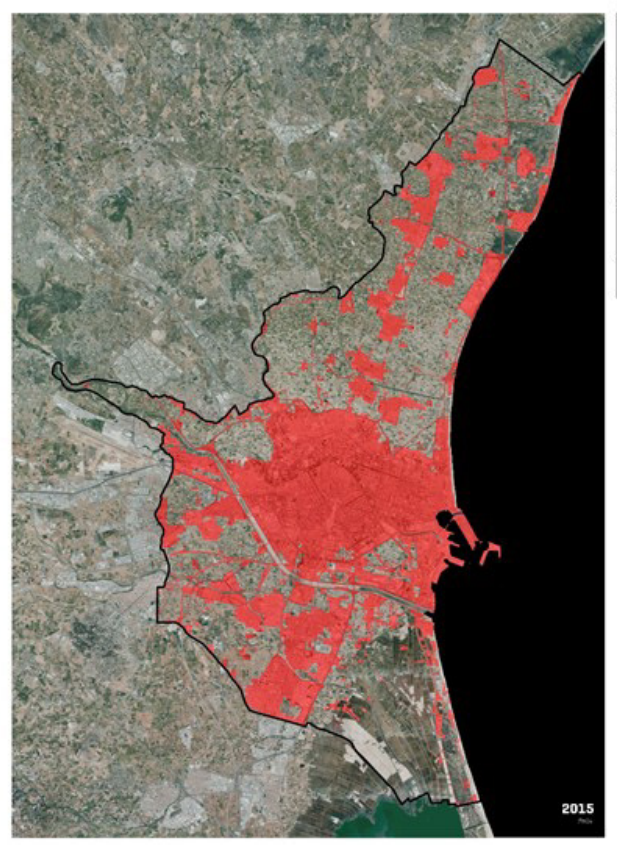

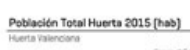
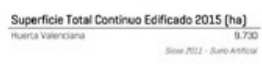

Las huertas históricas periurbanas del Mediterráneo 1:100.000 Mapa de la Huerta Histontorica de Valencia. Comparativa 1957- 2015

Fig. 8. Comparación de ocupación del suelo de la huerta de Valencia. 1957-2015. Fuente: Elaboración propia. 
núcleo del Grao y Poblados Marítimos junto al puerto y toda una constelación de pequeños asentamientos dispersos por la huerta que por su tamaño parecen estar claramente vinculados con la economía agrícola.

La realidad de 2015 evidencia otra forma urbana de la ciudad de Valencia, claramente informe, con la absorción completa de los asentamientos del litoral y un crecimiento extensivo que ha dejado sólo dos bolsas de huerta sin colmatar: Horta de Campanar al noroeste y Horta de Rovella i Francs, Marjals i Extremals a sureste. A esto se le une toda una constelación de geometrías y formas urbanas que producen el borde entre la ciudad y la huerta. Los municipios situados en la huerta también han experimentado crecimientos diversos que en algunos casos han llegado a producir conurbaciones interrumpiendo la continuidad de la huerta. Se aprecia una

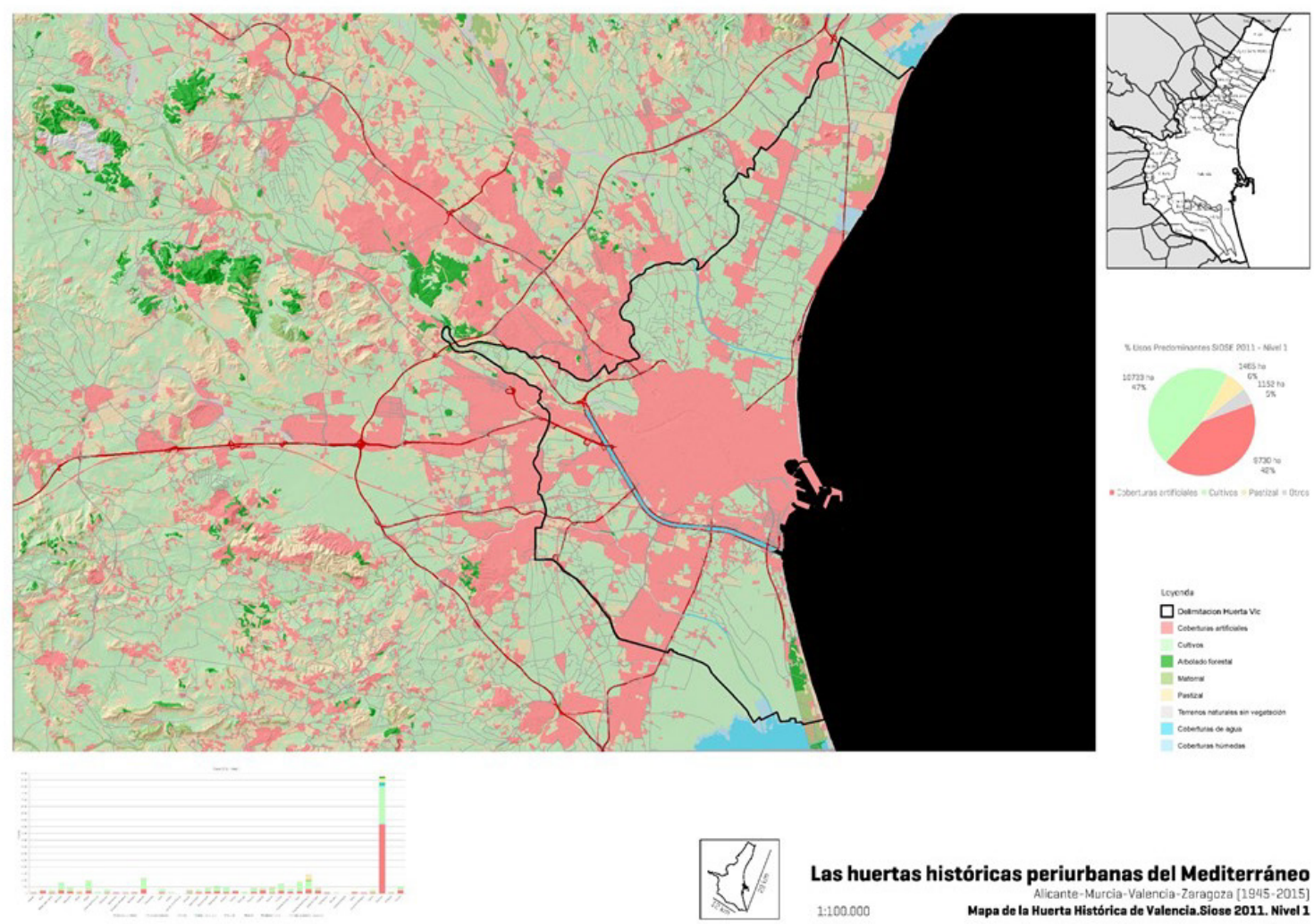

Fig. 9. Ocupación del suelo de la huerta de Valencia. Siose. Nivel 1. Fuente: Elaboración propia.

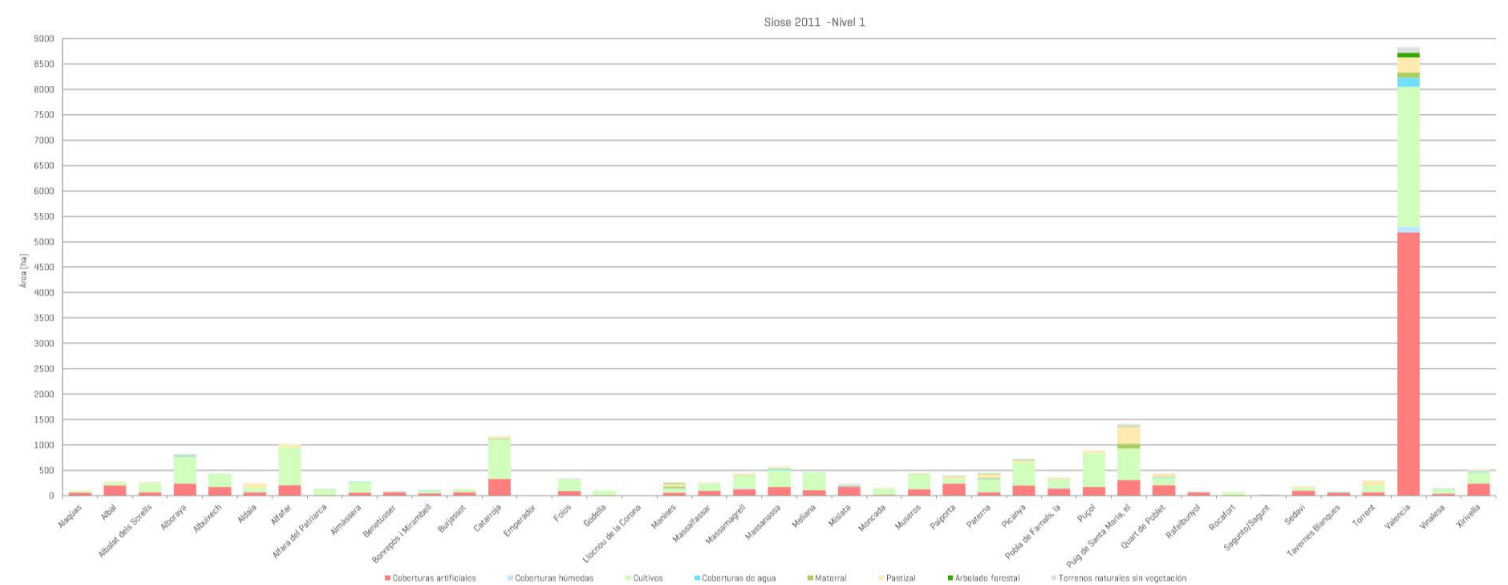

Fig. 10. Gráfico de representación de ocupación del suelo por municipios. Fuente: Elaboración propia. 
importante estructura lineal de este tipo en la huerta norte, apoyada en la antigua carretera nacional a Barcelona, y en el sur el conjunto de municipios Sedaví, Alfafar, Benetússer, Massanassa, Catarroja..., identificados por la gran presencia de suelo industrial. Esto deja patente la fractura de un territorio agrícola continuo que en 1957 todavía guardaba un equilibrio entre lo construido y lo no construido. En 2015, la huerta sobrante se reconoce en el sur por bolsas o islas mientras que en el norte todavía conservan, en gran parte, su continuidad. Por tanto, uno de los cambios topológicos más relevantes en este intervalo es el proceso de fragmentación o gestación de un "archipiélago" formada por continuos edificados y separados por claros "arrecifes" polarizados en torno a los corredores infraestructurales.

Valoración de usos a partir del Siose 2011. Nivel 1

Mirando con detenimiento la cartografía de ocupación del SIOSE. Nivel 1, se aprecia como lo construido, basándose en las coberturas artificiales, se aproxima a unas cifras semejantes a las de las áreas cultivadas dentro del ámbito estricto de la huerta: esto es, $42 \%$ y $47 \%$ respectivamente. Podemos decir que se ha producido un crecimiento (o decrecimiento de la huerta) que tiende a igualar lo construido con lo agrícola, lo que deja patente el cambio de situación que deben conducirnos a una reflexionar sobre el modelo de crecimiento urbano que hemos venido teniendo durante los últimos 50 años. (Figura 9)

Un análisis especifico por municipio, dejando de lado el caso concreto de la ciudad de Valencia por su singularidad, nos muestra que gran parte de los municipios dentro del ámbito de la huerta guardan un equilibrio razonable en cuanto a lo que es la superficie de su término municipal y la destinada a superficie de cultivo, esto es, huerta respecto al total. Esto podría servir de referencia para un potencial indicador dentro de la investigación que destacara aquellos municipios que continúan siendo agrícolas o que se pueden considerar "reservas de huerta" para el conjunto (Figura $10)$.
Valoración de usos a partir del Siose 2011. Nivel 2

El análisis de este nivel de leyenda deja entrever algunos aspectos particulares en cuanto a las áreas de cultivo. El total de áreas agrícolas asciende a un $54 \%$ del suelo, pero al valorar la diferencia de ocupación del suelo entre los cultivos herbáceos (33\%) y cultivos leñosos $(21 \%)$ se aprecia que la cifra no es insignificante con la que la presencia de los cultivos leñosos parece que ha ido creciendo en el tiempo. Esto por un lado implica la sustitución de los cultivos herbáceos, base del paisaje de la huerta, pero por otro significa la construcción de un nuevo paisaje que convive con el original. Esta reflexión es de especial interés pues supone un indicador claro del cambio de paisaje que debe ser estudiado con mayor profundidad en el transcurso de la investigación (Figura 11).

El territorio que queda circunscrito dentro del ámbito de la huerta histórica se identifica por una gran variedad de paisajes soportado en gran parte, por la naturaleza diversa de los tipos de cultivo, la posición geográfica (litoralinterior), las estaciones, los tipos de suelo, etc. que cada uno de ellos implica. De hecho, el Plan de Acción Territorial de la Huerta de Valencia (PATH), cuando analiza este paisaje identifica diferentes Unidades de Paisaje (UP), en concreto un total de 24 . Reconociendo dichas singularidades dentro de este gran ámbito, las UP's puede servir de recintos de referencia en la investigación que nos faciliten estudiar fenómenos concretos propio de ámbitos más pequeños. Estos aspectos deben ser abordadas en fases posteriores.

\section{Valoración de usos. Siose 2011. Suelo Artificial}

Esta cartografía describe la composición del suelo artificial lo que identifica la diversidad de morfologías, usos y tamaños de la transformación del suelo de la huerta en suelo continúo construido. El mosaico de colores claramente revela como en este periodo de 70 años la huerta ha ido sustituyéndose progresivamente por áreas destinadas a zonas de expansión urbana (ensanche en la nomenclatura 
del Siose) y áreas industriales principalmente. Las huertas alrededor de los cascos históricos de los municipios se han transformado intensamente con los crecimientos por extensión $(40 \%)$ y la presencia de polígonos, áreas industriales (14\%) y de dotaciones. De hecho, estos lugares son similares y genéricos en todos los municipios llegando a la idea de los lugares atópicos (Boeri, Lanzani \&, Marini, 1993). Cabe destacar que el crecimiento urbano discontinuo disperso ha sido muy pequeño (3\%) no desvirtuando con ello el espacio agrícola. Esta situación es claramente diferente en otros ámbitos de huerta como la de Murcia, objeto de nuestra investigación conjunta.

La incorporación sobre la huerta de usos tan diversos como los mencionados durante este periodo de tiempo, ha acarreado la aparición de infraestructuras de comunicación y de servicios, generando un sin fin de conflictos visuales, sonoros, de contaminación trasformando las dinámicas propias de lo que era la huerta histórica para pasar a ser un ámbito agrícola complejo en un entorno ya metropolitano.
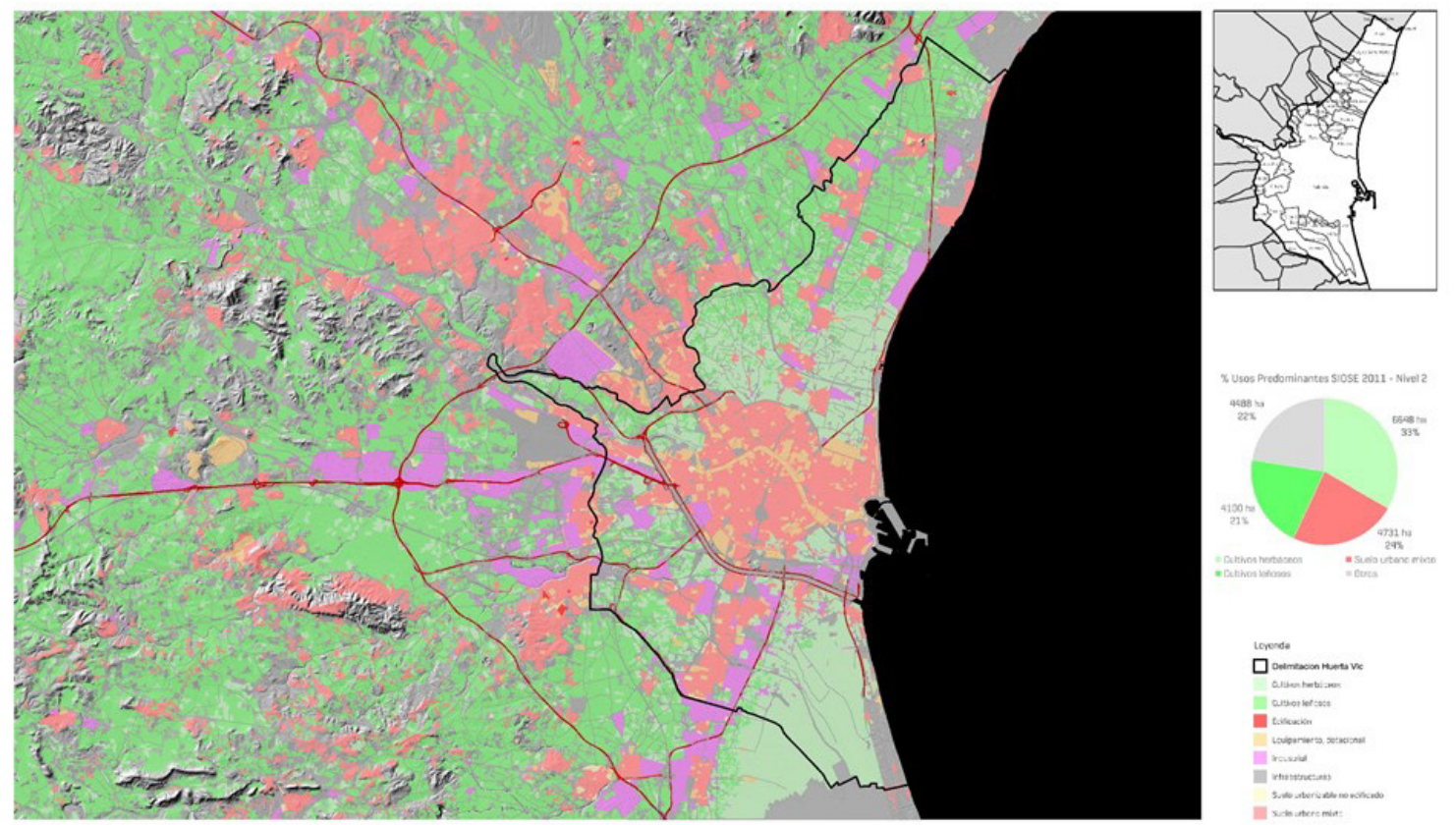

Las huertas históricas periurbanas del Mediterráneo $1: 1000000$

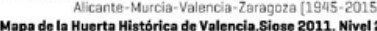

Fig. 11. Ocupación del suelo de la huerta de Valencia. Siose. Nivel 2. Fuente: Elaboración propia.

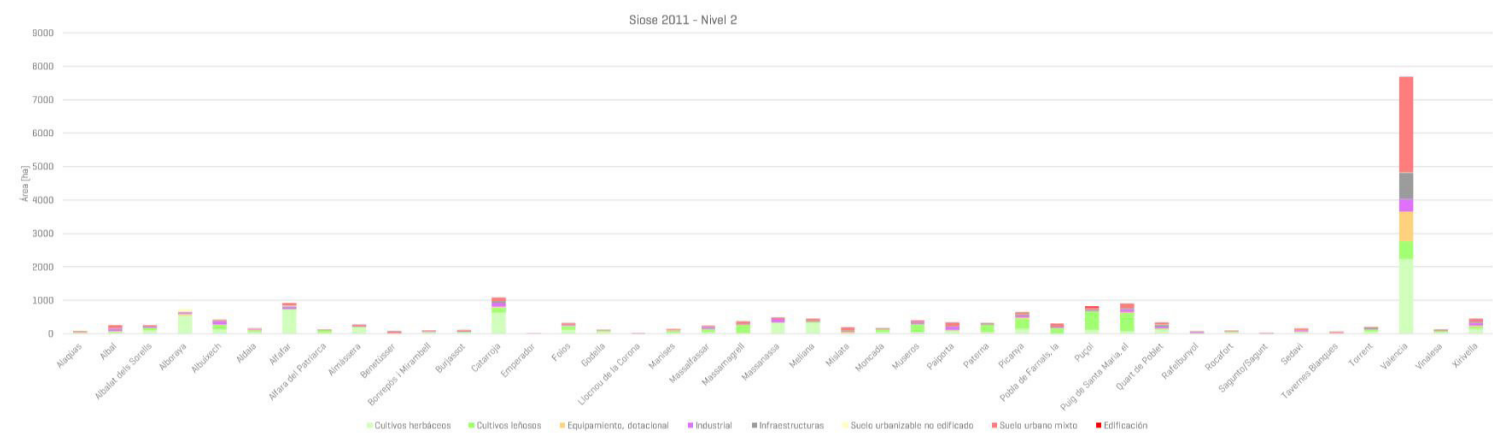

Fig. 12. Gráfico de representación de ocupación del suelo por municipios. Fuente: Elaboración propia. 
Mapa de la Huerta Histórica de Valencia. Siose 2011. Suelo Cultivos

El análisis del suelo de cultivos evidencia como la situación presente guarda una proporción en la que cultivos leñosos (cítricos) marcan un $37,9 \%$ y los cultivos herbáceos un $43,2 \%$. Estas cifras dejan patente la progresiva sustitución del suelo de la huerta tradicional por cultivos de cítricos de regadío. El mapa representa que las áreas destinados al cultivo de cítricos no son las regadas por las acequias históricas, si por pozos que han permitido la ampliación y mejora de los campos de cultivo, el tratamiento y el ahorro del agua. Esto refuerza la clara transformación del paisaje dentro del ámbito de la huerta histórica en una convivencia desigual de áreas de herbáceas, cítricos y arrozales. Esto puede arrastrar a una posible confusión (sino sustitución) de la huerta con el huerto.

El mapa indica también el valor ambiental que las áreas de huerta conservan y, por tanto, muestran el grado de protección que deben recibir (el PATH ya lo establece). Analizar con detenimiento este paisaje cultural de huerta, reconocer su estructura e imbricación con lo urbano puede hacer pervivir la huerta frente a su sustitución por otros cultivos ajenos a los herbáceos o por los suelos artificiales.

\section{Conclusiones}

Como afirma Pérez-Campaña (2013), los espacios periurbanos son espacios de conflictos marcados por un crecimiento urbano e infraestructural y una dinámica progresiva de debilitación del sector agrario, resultando especialmente compleja la tarea de compatibilización de usos en territorios que son a la vez espacios rurales y áreas de influencia urbana (Menor Toribio, 2000). Esta influencia urbana es resultado del proceso de periurbanización que conlleva la progresiva transformación del paisaje en las proximidades de los núcleos urbanos, modificando su estructura y apareciendo nuevos elementos propios de la estructura urbana o suburbana (Antrop, 2004). El proceso de periurbanización conlleva la dispersión de la ciudad en el territorio lo que puede llevar a la retirada progresiva de la agricultura y al abandono de tierras (Entrena Durán, 2004), la pérdida de estructuras

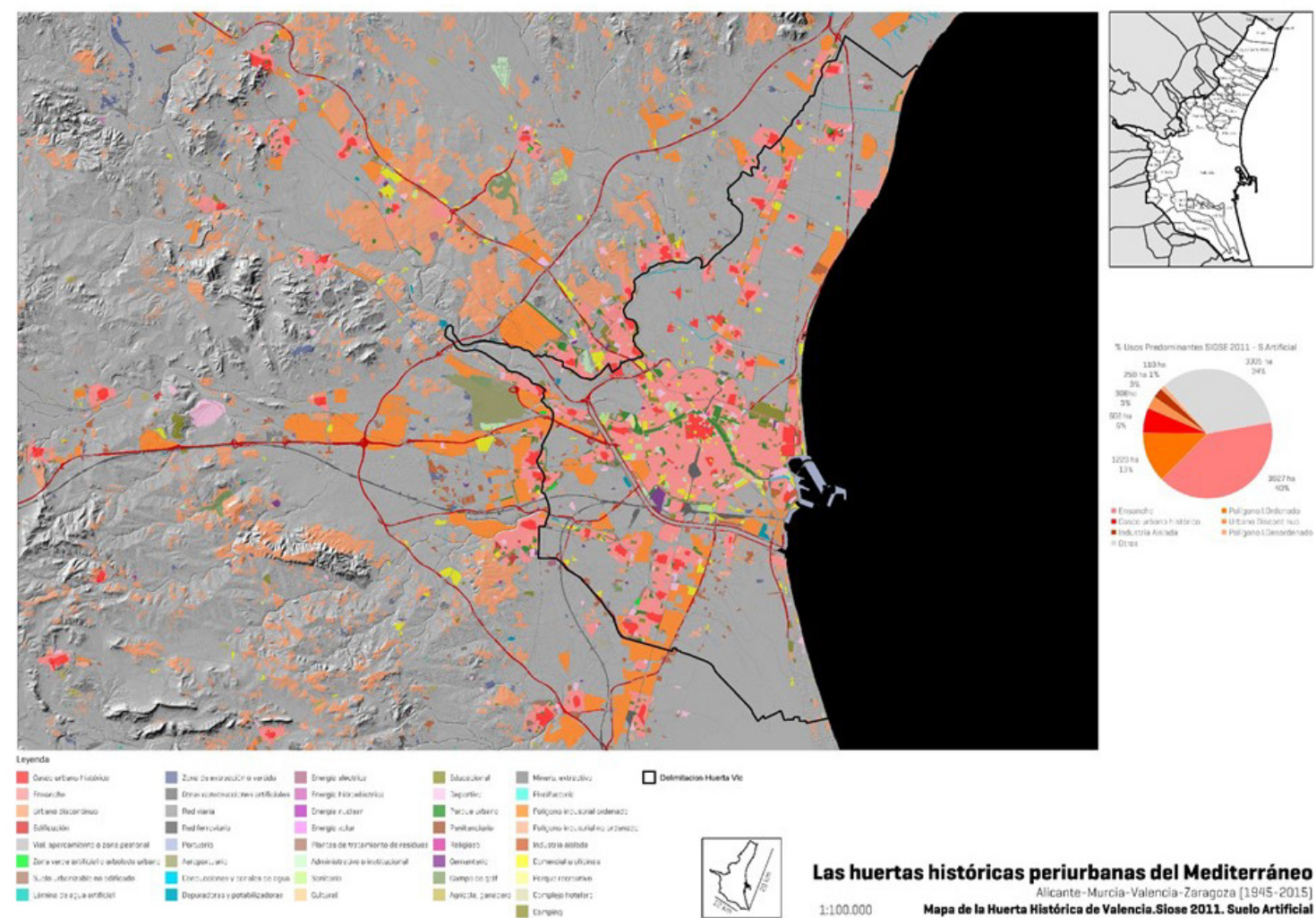

Fig. 13. Ocupación del suelo de la huerta de Valencia. Siose. Suelo artificial. Fuente: Elaboración propia. 
históricamente muy elaboradas del paisaje rural (Dematteis, 1998), un aumento de las necesidades de movilidad (Fariña Tojo, 2003; Rueda, 2003) y, en definitiva, el deterioro de la calidad del espacio. Hablamos de una crisis de los paisajes rurales y periurbanos (Donadieu y Luginbühl, 2008) resultado también de una crisis de la agricultura mediterránea (Campos Climent, 2011)

Este trabajo de investigación conjunta, que aglutina las visiones de especialistas en cada uno de los espacios de huerta periurbana, trabaja en la elaboración de estrategias de integración y salvaguarda de estos espacios a partir de una mejor comprensión y parametrización de los espacios de huerta más resilientes. En esta aproximación comparativa se han experimentado métodos que permitan solventar las disparidades en las bases de datos cartográficas de usos del suelo de los distintos espacios, muestra de la falta de criterios eficientes para la clasificación de este tipo de espacios agrícolas periurbanos.
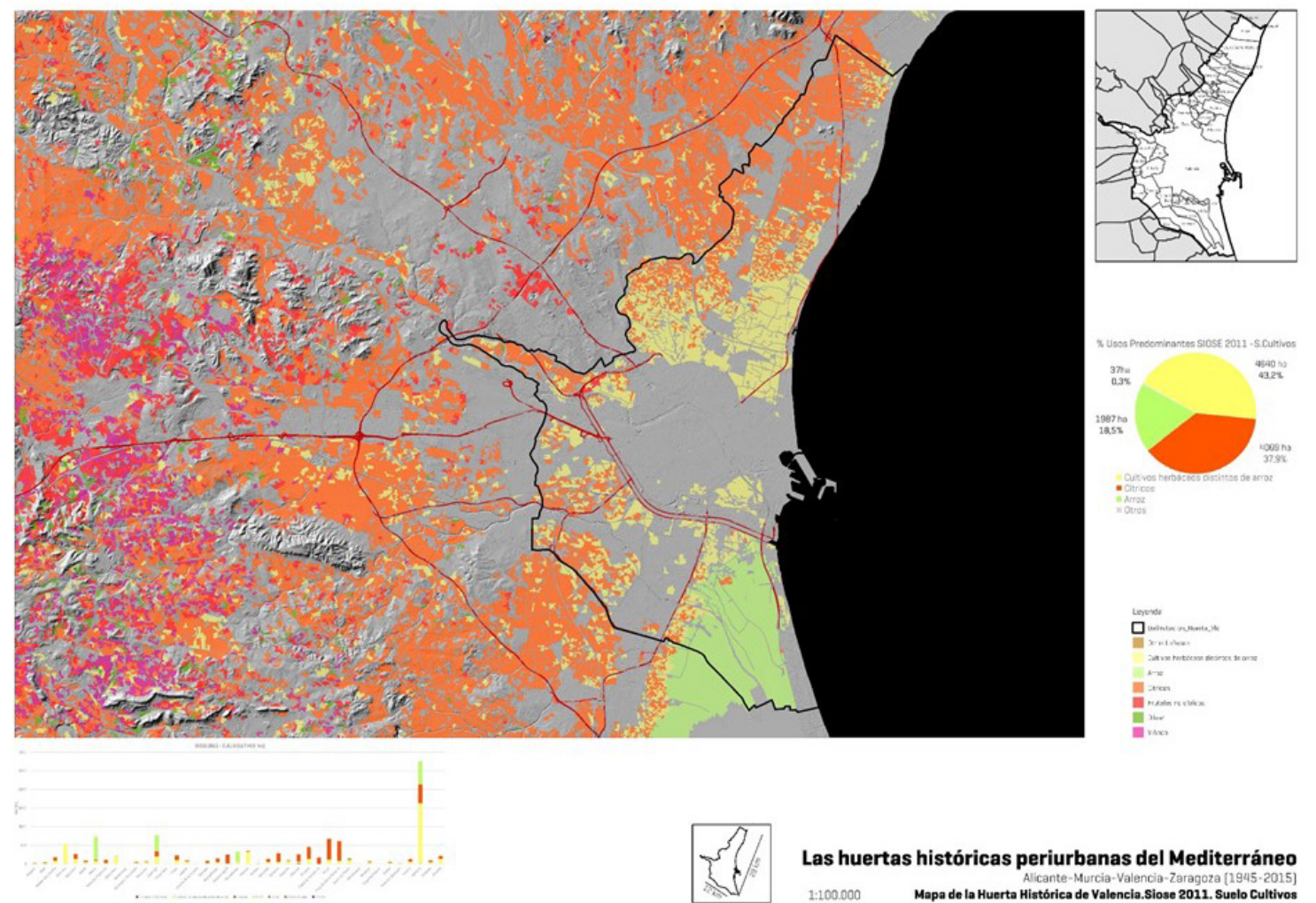

Las huertas históricas periurbanas del Mediterráneo 1:100.000 Mapa de la Huerta Historice-Murcia-Valencia-Zaragoza [1945-2015]

Fig. 14. Ocupación del suelo de la huerta de Valencia. Siose. Suelo de cultivos. Fuente: Elaboración propia.

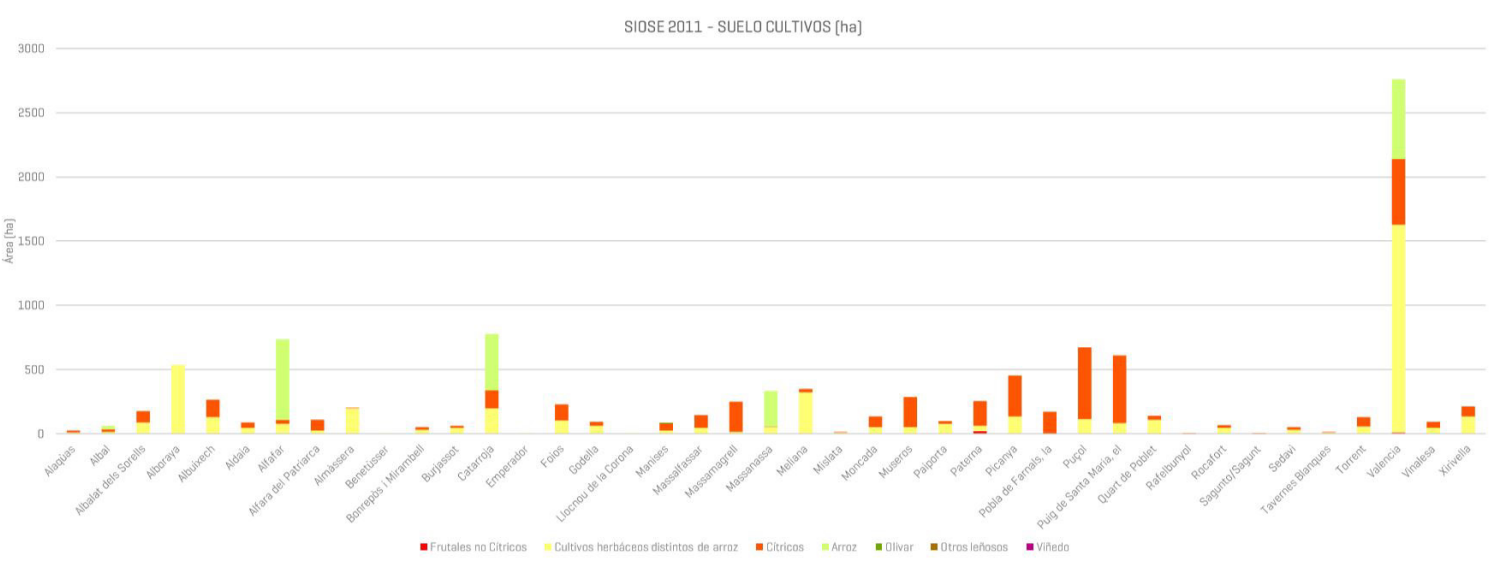

Fig. 15. Gráfico de representación de ocupación del suelo por municipios. Fuente: Elaboración propia. 


\section{Agradecimientos}

Agradecemos a la Universidad Politécnica de Cartagena y la Universidad San Jorge de Zaragoza las ayudas concedidas para la celebración de la I y II Jornada de Investigación sobre Huertas Históricas Periurbanas del Mediterráneo.

\section{Notas al pie}

1 Red de investigación formada por investigadores de las siguientes universidades: Universidad Politécnica de Cartagena; Universidad de Alicante; Universitat Politènica de València; Universidad San Jorge, Zaragoza

2 I Jornada de Investigación bajo el título "Las huertas históricas periurbanas del mediterráneo: Alicante-Murcia-ValenciaZaragoza" celebrada el 9 de marzo de 2018 en la UPCT (https://www.upct.es/destacados/ cdestacados.php?ubicacion=general\&id buscar $=8970$ ). II Jornada de Investigación bajo el título "Huertas históricas periurbanas del mediterráneo: Alicante-MurciaValencia-Zaragoza" celebrada el 27-28 de junio de 2019 en la Universidad San Jorge, Zaragoza. (https://aos.usj.es/post/2019_06_ jornadahuertas/)

\section{Referencias}

Antrop, M. 2004. "Landscape change and the urbanization process in Europe", Landscape and Urban Planning, 67(1-4): 9-26. doi. org/10.1016/S0169-2046(03)00026-4.

Beltrán, J. P. 2012. "El desafío global de la producción de alimentos y l'Horta de Valencia”. En: J. Romero \& M. Francés (Eds) La Huerta de Valencia. Un Paisaje Cultural con Futuro Incierto, pp. 173-196 (Valencia: PUV).

Boeri, S., Metropolitani, A. I., Lanzani, A., \& Marini, E. 1993. Il territorio che cambia: ambienti, paesaggi e immagini della regione milanese. Abitare Segesta.

Burriel, E. 1971. "La Huerta de Valencia, Zona Sur". Estudio de Geografía Agraria.Valencia: Institución Alfonso el Magnánimo.

Campos Climent, V. 2011. La crisis de la agricultura mediterránea en el Arco Mediterráneo Central. Un estudio sobre sus causas, propuestas de solución y fi nanciación desde la economía social. Universidad de Valencia, Valencia.

Courtot, R. 1986. Agriculture irriguée et organisation ou l'espace dans les huertas de Valencia et de Castellon. Paris: Université Paris VII.

Dematteis, G. 1998. "Suburbanización y periurbanización. Ciudades anglosajonas y ciudades latinas". En: Monclús, F.J., Ed. La ciudad dispersa. Barcelona, Centro de Cultura Contemporánea, 17-33.

Donadieu, P. y Luginbühl, Y. 2008. "Il divenire dei paesaggi rurali e peri-urbani". En: Donadieu, P., Küster, H., Milani, R., (ed.). La cultura del paesaggio in Europa tra storia, arte e natura. Manuale di teoría e pratica Firenze, Leo S. Olschki, 157-180.

Entrena Durán, F. 1998. Cambios en la construcción social de lo rural: de la autarquía a la globalización. Tecnos, Madrid.

Fariña Tojo, J. 2003 "Sostenibilidad y racionalidad de los procesos de urbanización". En: Arenillas, T. Coord. Ecología y ciudad: raíces de nuestros males y modos de tratarlos. Fundación de Investigaciones Marxistas, Madrid.

García Martín, F.M. y Ros Sempere, M., 2016b. "Patrones de ocupación informal del territorio periurbano de la Huerta de Murcia, 1929-2015”. En: FUNDICOT - Asociación Interprofesional de Ordenación del Territorio (ed.), VIII Congreso Internacional de Ordenación del Territorio. Nuevos tiempos, nuevos objetivos. Fuerteventura. Relatoria, ponencias y comunicaciones [en línea]. Fuerteventura: s.n., pp. 465-477. Disponible en: http://www.fundicot.org/\#!viii-ciot-du/ i90e6.

García Mayor, C., \& Canales Martínez, G. (2015). La huerta de Orihuela en el Bajo Segura: elementos funcionales en la configuración del paisaje. (Universidad de Alicante, Ed.). Alicante: Universidad de Alicante. http://rua.ua.es/dspace/ handle/10045/52734

Garcia-Mayor, C. (2015). Territorio, paisaje e identidad. La Huerta de Orihuela en la Vega Baja del río Segura. Universidad de Alicante. 
http://rua.ua.es/dspace/handle/10045/85324

García-Mayor, C. (2017). Invariants in the evolution and territorial identity of the traditional Huerta landscape of the Vega Baja of the Segura River: 1929-2010. Boletin de La Asociacion de Geografos Espanoles, 2017(73), 369-388. https://doi. org/10.21138/bage. 2422

Garcia-Mayor, C., \& Canales Martínez, G. (2018-a). Poly-nuclear urban system, landscape identity and economic development: The Vega Baja of the Segura River (Alicante) case study. In D. Urios, J. Colomer, \& A. Portalés (Eds.), 24th ISUF International Conference. City and territory in the Globalización (pp. 47-53). Valencia: Editorial Universitat Politècnica de València. https://rua.ua.es/dspace/ handle/10045/75990

García-Mayor, C., \& Canales Martinez, G. (2018-b). La Vega Baja del Segura, una huerta sitiada: la transformación del territorio. Cátedra Arzobispo Loazes, Universidad de Alicante. https://rua.ua.es/ dspace/handle/10045/88415

García-mayor, C., Miguel, F., Martín, G., \& Sempere, M. R. (2018). Huertas periurbanas de las Vegas del Segura: un proceso de fragmentación / dispersión del paisaje productivo. In BIA Urban Regeneration Forum. Bilbao.

Generalitat Valenciana. 2016. Plan de Acción Territorial de Ordenación y Dinamización de la Huerta de Valencia. Valencia, Conselleria d'Habitatge, Obres publiques i Vertebració del Territori.

Generalitat Valenciana. 2017. Web de Descargas de Cartografía del Instituto Cartográfico Valenciano (Terrasit). http://terrasit.gva. es 10/09/2017, Valencia, Departamento de Información de Base y Cartografía.

Glick, T.F. 1970. Irrigation and Society in Medieval Valencia.Cambridge: Cambridge University Press.

Lovell, S. 2009. "Designing Landscapes for Performance Based on Emerging Principles in Landscape Ecology". Ecology and Society, 14 (1),44 (online)

Menor Toribio, J. 2000. La Vega de Granada: transformaciones agrarias recientes en un espacio periurbano. Universidad de
Granada, Granada.

Pérez Campaña, R. 2013. La vega del Guadalfeo como paisaje agrario periurbano: transformación, ecoestructura y multifuncionalidad. Granada: Universidad de Granada, 348 p. http://hdl.handle. net/10481/29816

Romero, J., \& Melo, C. 2016) La ordenación y gestión de las huertas mediterráneas españolas. El tiempo de la (s) política (s). En: Vera, J. Fernando; Olcina, Jorge; Hernández, María (eds.). Paisaje, cultura territorial y vivencia de la Geografía. Libro homenaje al profesor Alfredo Morales Gil. San Vicente del Raspeig: Publicaciones de la Universidad de Alicante, 2016. ISBN 978-84-16724-03-1, $1202 \mathrm{p}$

Ros Sempere, M. y García Martín, F. M. 2016a. Cinco palmos 1929-2015. Transformación de usos del suelo en la Huerta de Murcia. CRAI Biblioteca, Universidad Politécnica de Cartagena, 93 pp.

Ros Sempere, M. y García Martín, F.M., 2017. Stages of terrritorial configuration in the nonplanned occupation of the Huerta de Murcia, 1929-2015. En: 24th ISUF International Conference, City and territory in the globalization age. Conference proceedings [en línea]. Valencia: Universidad Politécnica de Valencia, pp. 97-106. DOI http://dx.doi. org/10.4995/ISUF2017.2017.6281 2017, Disponible en: http:/ocs.editorial.upv. es/index.php/ISUF/ISUF 2017/paper/ view/6281.

Rueda, S. 2003. "Modelos de ordenación del territorio más sostenibles". En: Calvo López, R. (ed.). La sostenibilidad en el proyecto arquitectónico y urbanístico. Ciudades para un futuro más sostenible, Madrid.

Temes Cordovez, R.; Moya Fuero, A. 2016. "Typology of the transformations occurred in the peri-urban space of Huerta de Valencia. Evidence from North arch of Valencia (Spain)". International Journal of Sustainable Development and Planning. 11(6):996-1003. doi:10.2495/SDPV11-N6-996-1003

Temes Cordovez, RR. 2008 . "Las fuentes catastrales y la identificación de las transformaciones en los tejidos urbanos". Revista CT Catastro n ${ }^{\circ} 64$ 
Temes Cordovez, RR.; Moya Fuero, A. 2015.

"Dynamics of change in the peri-urban landscape of Huerta de Valencia: the case of La Punta (Valencia)". WIT Transactions on Ecology and the Environment. 192:123-131. doi:10.2495/ECO150121

Tuset, J. 2017. "Green Coastal Zones: Nodes and connectors as strategy of urban regeneration". International Journal of Sustainable Development and Planning, 12(3).488-497

Tuset, J. J., Temes, R., \& Ruiz, A. 2018. Huertas periurbanas en Valencia y Zaragoza : modelo de paisaje multifuncional en la Huerta de Campanar. En: BIA Urban Regeneration Forum. Bilbao. 